\title{
LA AUTOESTIMA Y SU RELACIÓN CON LA PERCEPCIÓN DEL APRENDIZAJE: EL CASO DE LOS ESTUDIANTES DE ARQUITECTURA DE LA UNIVERSIDAD AUTÓNOMA DE NUEVO LEÓN
}

\author{
Irma Laura Cantú Hinojosa \\ Universidad Autónoma de Nuevo León, México
}

\begin{abstract}
RESUMEN
Mediante un estudio descriptivo y correlacional se procuró determinar la asociación que existe entre la autoestima y el aprendizaje percibido por los alumnos de arquitectura de la UANL. En un muestreo probabilístico estratificado por semestres se seleccionaron 388 sujetos de una población total de 1153 estudiantes de arquitectura, a quienes se administraron dos instrumentos: uno, elaborado ex profeso para esta investigación, midió la variable aprendizaje percibido, mientras el otro determinó el nivel de autoestima de cada estudiante. Se halló el coeficiente $\mathrm{r}$ de Pearson para determinar la correlación de la autoestima con el aprendizaje percibido por el grupo de estudiantes universitarios de la muestra. Los resultados muestran que la autoestima correlacionó significativamente con el aprendizaje percibido por los estudiantes del Nivel Medio en las áreas curriculares de Tecnología y en la de Formación General. También en el Nivel de Integración en las áreas curriculares de Diseño y en la de Formación General. No se encontró correlación en el Nivel Básico en ninguna de las áreas curriculares del mismo programa educativo.
\end{abstract}

Palabras clave: autoestima, aprendizaje percibido, estudiantes de arquitectura

Introducción

En los diferentes niveles educativos, la autoestima de los estudiantes ha sido asociada con diversos factores que rodean las condiciones en que ellos logran el aprendizaje. Cotton (1989) afirma que el aprendizaje es un proceso de adquisición de un nuevo conocimiento y habili-

Irma Laura Cantú Hinojosa, Facultad de Arquitectura, Universidad Autónoma de Nuevo Léon, Monterrey, Nuevo León, México.

La correspondencia concerniente a este artículo puede ser enviada a Irma Cantú, Loma Bonita 212, Col. Loma Larga, Monterrey, Nuevo León, México 64710. Correo electrónico: irmacantu@intercable.net dad; menciona que para que este proceso pueda ser calificado como aprendizaje, en lugar de una simple retención pasajera, debe implicar una retención del conocimiento o de la habilidad en cuestión, de tal forma que permita su manifestación posterior. Según algunas teorías, la autoestima condiciona que el mismo estudiante formule una construcción mental nueva o que revise una previa (conocimientos conceptuales como actitudes y valores) (Abbagnano, 1994).

El aprendizaje conduce a cambios de larga duración en el comportamiento potencial. Este concepto se refiere al comportamiento posible (no necesariamente actual) de un individuo en una 


\section{CANTÚ HINOJOSA}

situación dada para poder alcanzar una meta. Sin embargo, el solo potencial no es suficiente: el aprendizaje necesita ser reforzado para que perdure (Bruner, 1996).

Son muchas las variables que afectan al aprendizaje y el rendimiento académico de los alumnos. Las hay de tipo personal, familiar, ambiental y de contexto (Beltrán, 1987). La capacidad de aprendizaje está normalmente restringida por las características psicológicas de la percepción, la comprensión y del pensamiento humano.

Ante las implicaciones de conceptos tan disímiles, lo que sí parece claro es que los alumnos que fracasan, tanto por rendir menos de lo que pueden como los que simplemente no rinden, presentan actitudes hacia sí mismos y sus potencialidades intelectuales profundamente negativas (Coll, 1992). Se perciben menos capaces, desajustados y menos confiados que los compañeros que tienen éxito. De aquí se puede extraer la idea de que, en general, todo el material y las estrategias didácticas que utilice el profesor con la intención de mejorar el autoconcepto del estudiante estará de alguna manera sentando las bases para mejorar su rendimiento futuro.

Ante la pregunta $¿$ Es el aprendizaje y el rendimiento académico lo que origina un buen autoconcepto o, por el contrario, es el autoconcepto el responsable de la mejora del rendimiento y el aprendizaje? La mayoría de las investigaciones apuntan a la existencia de relaciones persistentes y significativas entre ambos conceptos. La relación parece tener naturaleza bidireccional con una interacción recíproca y continua entre ambas variables (Trianes, 1996).

Debido a lo anterior, los profesionales de la educación cada vez son más conscientes de la importancia del autoconcepto y la autoestima y de su estrecha relación con el aprendizaje y el rendimiento escolar de los alumnos (Román Pérez y Díez López, 2000). El concepto de autoestima ha tenido papeles preponderantes en la toma de decisiones en ciertos sistemas educativos (Melendo, 2006). A principios de la década de los $90^{\prime}$, en los Estados Unidos y otros países anglosajones, la autoestima se convirtió en un concepto en boga entre algunos teóricos de la pedagogía. La teoría propuesta era que la autoestima es una causa de las actitudes constructivas en los individuos, y no su consecuencia, diciéndose que si, por ejemplo, un estudiante tiene buena autoestima, entonces tendría buenos resultados académicos $\mathrm{y}$, por lo tanto, un eficiente desempeño profesional.

En este contexto, la autoestima puede definirse como una fuerza interna que impulsa a la persona a desarrollarse, a poner en práctica sus capacidades, de manera que se vaya orientando hacia un equilibrio personal, y en ocasiones puede ser causa y consecuencia de la manera en que se comportan las personas en distintas situaciones de la vida (Ribeiro, 2006). Se relaciona con el hecho de estar conscientes de las propias potencialidades y necesidades (Harter, 1999). En este sentido, la educación se enfrenta a un nuevo desafío: lograr que los estudiantes desarrollen una sana autoestima, que les permita potencializar sus capacidades, conocimientos, habilidades y en general el aprendizaje a lo largo de sus estudios.

Según Beltrán (1987), la autoestima está formada por sentimientos aprendidos y pensamientos positivos que reflejan una actitud positiva de "puedo hacerlo" contra una actitud pesimista de "no puedo hacerlo". La autoestima saludable es una evaluación realista de las propias 
capacidades y tiene sus raíces en el respeto merecido de los demás (Skaalvik y Hagtvet, 1990). Para Coopersmith (1994), la autoestima es un juicio personal de dignidad, que se expresa en las actitudes del individuo hacia sí mismo. Es una actitud de aprobación o desaprobación e indica en qué medida el individuo se cree capaz, importante, digno y con éxito. Bednar, Wells y Peterson (1995) ofrecen otra definición de autoestima, concibiéndola como un sentido subjetivo de autoaprobación realista. Desde esta perspectiva, la autoestima es fundamentalmente un sentido perdurable y afectivo del valor personal, basado en una autopercepción exacta, que implica competencia, seguridad, dominio, logro, independencia y libertad.

En sus investigaciones, Palladino (1994) destaca el aspecto de confianza en sí mismo que se da en una autoestima sana y afirma que la autoestima es el aprecio del valor e importancia propios y la posesión del carácter para ser responsable de sí y de actuar responsablemente hacia los demás.

Relacionado con el concepto de autoestima está el de autoconcepto. El autoconcepto es un fenómeno complejo, un constructo que incluye las imágenes de lo que creemos ser, lo que deseamos ser y lo que presentamos o queremos presentar a los demás. Es un conjunto de percepciones organizado, jerárquico, coherente y estable, aunque también susceptible de cambios. Es una organización cognitivo-afectiva que influye en la conducta. Incluye diversas dimensiones: la académica, social, emocional y física (Shavelson y Bolus, 1982; Marsh 1986).

Hay algunos estudios centrados en cómo facilitar el desarrollo del autoconcepto en la escuela para impulsar el rendimiento académico y el aprendizaje
(Boersma y Chapman, 1985; Combs, 1974; Harter, 1982, 1985a, 1985b; Marsh, 1988; Purkey, 1970; Purkey y Novak, 1984). Los estudios de Byrne y Gavin (1996) prestan mayor atención a los aspectos estructurales del autoconcepto (sus componentes cognitivos) y no sólo a sus elementos evaluativos y afectivos (autoestima). Hay otros estudios que asocian el autoconcepto con el rendimiento escolar y el aprendizaje (Burns, 1980, 1990; García Torres, 1983; Markus y Nurius, 1986; Marsh, 1986, 1990b; Marsh, Parker y Smith, 1983; Oñate y García, 1995; Shavelson y Bolus, 1982; Shavelson, Hubner y Stanton, 1976).

En otras investigaciones se ha encontrado relación entre el autoconcepto y los logros escolares de los alumnos. Aún así, los resultados de las investigaciones más relevantes no son concluyentes. Marsh (1990a) encuentra que el autoconcepto determina causalmente el rendimiento del alumno, mientras que Chapman y Lambourne (1990) sostienen lo contrario. Skaalvik y Hagtvet (1990) obtienen una relación recíproca. Por su parte, Helmke y van Aken (1995) incrementan la incertidumbre en su afirmación sobre la hipótesis de la influencia del rendimiento académico sobre el autoconcepto, aunque señalan una variación tomando en cuenta la edad de los sujetos.

Esta investigación se dirige hacia el papel de la autoestima en la percepción que tienen los estudiantes acerca de su propio aprendizaje.

McCombs y Whisler (1997) desarrollaron doce grandes principios del aprendizaje centrado en el alumno, entre los cuales se destaca la importancia de la motivación y la autoestima como factores que influyen en el proceso de aprendizaje.

Para Hernández (1991), las variables 


\section{CANTÚ HINOJOSA}

personales que determinan el aprendizaje escolar, son la inteligencia (el poder), la motivación (el querer) y la personalidad (modo de ser). Goodwin (1999) señala que el nivel de autoestima es el responsable de muchos éxitos y fracasos escolares.

Otras investigaciones también muestran que, cuando los alumnos hacen conexiones entre su propia identidad y la institución educativa en donde estudian, estas conexiones incitan el aprendizaje de por vida y el desarrollo de habilidades (McCombs, 1991; McCombs y Marzano, 1990). El reto de la enseñanza en el futuro implica el aprender a comprender y aplicar en la didáctica aspectos emocionales frecuentemente olvidados, tales como la autoestima, el autoconcepto y la motivación.

El desempeño escolar positivo pasa a ser el motivo de la autoestima y de la mejora de la autoimagen (Covington, 1984). En consecuencia, el alumno se atribuye a sí mismo los éxitos escolares (esfuerzo y capacidad) fortaleciendo su autoestima, mientras que adjudica sus fracasos a causas externas (falta de esfuerzo, empleo inadecuado de estrategias), evitando que afecten su autoestima. Los profesores, con la enseñanza, evaluación y valoración de los resultados de los alumnos, son también responsables del nivel de la autoestima que propician en ellos (Covington, 1992).

El papel de los maestros es muy importante, especialmente en las cuestiones referentes a la autoestima, porque el maestro espera conductas y aprovechamientos específicos de los estudiantes y debido a esas expectativas tiene un comportamiento propio con cada estudiante. Este trato hace saber al alumno qué conducta se espera de él y afecta su autoconcepto, motivación para el logro y nivel de aspiración. Si el trato es consistente y el alumno no se resiste, la interacción de ambos permite moldear el aprovechamiento y conducta del estudiante y a su vez el autoconcepto que éste tiene de sí mismo (Covington y Omelich, 1988).

Algunos otros estudios, como el de González-Pumariega Solís, García Rodríguez, García García, Núñez Pérez y González-Pienda García (1994), ponen de manifiesto que la implicación activa del sujeto en el proceso de aprendizaje aumenta cuando confía en sus propias capacidades y tiene altas expectativas de autoeficacia.

Es siempre importante la evaluación del sentimiento autoestimativo, dado que el nivel de autoestima podrá influir en muchos fracasos y/o éxitos académicos y personales. Una autoestima adecuada, vinculada a un concepto positivo, potenciará la capacidad del sujeto para desarrollar sus habilidades y aumentará el nivel de seguridad personal, mientras que una autoestima baja enfocará a la persona hacia la derrota y el fracaso.

El estudio aquí reportado tuvo por objetivo conocer la asociación de dependencia correlacional que existe entre la autoestima y el aprendizaje, a partir de la percepción que experimentan los estudiantes de arquitectura de la UANL de los diferentes niveles académicos -Básico, Medio y de Integración- y las áreas curriculares que los conforman: Diseño, Teórico-Humanística, Tecnología y Formación General Universitaria.

\section{Hipótesis}

Las doce hipótesis que se pusieron a prueba en esta investigación formulan la existencia de una relación entre la autoestima y el aprendizaje para cada nivel 
académico (Básico, Medio y de Integración), en sus diferentes áreas curriculares (Diseño, Teórico-Humanística, Tecnología y Formación General Universitaria).

\section{Método}

Se realizó un estudio cuantitativo, descriptivo y correlacional, utilizando un muestreo probabilístico estratificado que dividió la población de 1,153 estudiantes de arquitectura en estratos según el nivel de desarrollo académico, de acuerdo con el plan de estudio acreditado en agosto del 2006, considerándose la proporción adecuada para cada nivel. Se obtuvo una muestra total de 388 sujetos, de acuerdo con la fórmula de Hernández Sampieri, Fernández Collado y Baptista Lucio (2006) (ver Tabla 1).

Para conocer el nivel de aprendizaje

Tabla 1

Población y muestra estratificada según el nivel académico

\begin{tabular}{lcccc}
\hline \multicolumn{1}{c}{ Nivel } & Semestre & Población & Muestra & Totales \\
\hline Básico & 4 & 175 & 57 & 133 \\
& 5 & 246 & 76 & \\
\multirow{2}{*}{ Medio } & 7 & 225 & 76 & 131 \\
& 8 & 155 & 55 & \\
Integración & 9 & 217 & 72 & 124 \\
& 10 & 135 & 52 & \\
Total & & 1153 & 388 & 388 \\
\hline
\end{tabular}

-desde la percepción de los estudiantesse utilizó un instrumento realizado ex profeso para esta investigación, en la versión para cada nivel de estudio, que tiene una consistencia interna medida con un coeficiente alfa de .8998 para el del Nivel Básico, .9440 para el del Nivel Medio y .9422 para el del Nivel de Integración. Para la variable autoestima se utilizó un instrumento denominado "Test VA Grajales-Valderrama 2000", que presentó una consistencia interna medida con un coeficiente alfa de .8280 en toda la muestra. Se utilizó la prueba de dependencia $r$ de Pearson para estudiar la correlación del aprendizaje -desde la percepción del estudiante- con el nivel de autoestima.
La recolección de los datos fue realizada por la autora del estudio, quien administró los instrumentos después de conseguir la autorización de las autoridades académicas de la Facultad de Arquitectura de la Universidad Autónoma de Nuevo León. Se procedió a la elaboración de la base de datos en el programa estadístico SPSS, donde se realizaron los análisis para las pruebas de hipótesis correspondientes. Para la variable aprendizaje se calcularon cuatro escalas sumativas a partir de los valores de las respuestas que cada estudiante ofreció a cada uno de los indicadores del respectivo nivel académico. Para la variable autoestima, se siguió estrictamente lo manifestado en el instructivo que el mismo instrumento 


\section{CANTÚ HINOJOSA}

establece para su aplicación.

Resultados

Descriptivos

Los resultados descriptivos del comportamiento de las variables autoestima y aprendizaje percibido, según fue observado en los diferentes niveles académicos, se presentan en términos generales en las Tablas 2 y 3 .

Se observa, en particular, en la variable autoestima, que la media por niveles más baja fue de 30.86 , mientras la más alta fue de 31.56; sin embargo, al observarse el comportamiento de la misma variable en la muestra estratificada se aprecia que el grado de autoestima va cambiando a medida que avanzan los estudiantes a semestres superiores y se manifiesta en distintas áreas de estudio. En el Nivel Básico y el Medio, la mayoría de los estudiantes se presentan con una tendencia hacia los niveles altos de autoestima. En el Nivel de Integración, la distribución se dispersa; es decir, el nivel de autoestima de gran parte de los

Tabla 2

Datos descriptivos de la variable autoestima, según el nivel académico del programa académico de la carrera de arquitectura en la UANL $(N=388)$

\begin{tabular}{lccc}
\multicolumn{1}{c}{ Nivel académico } & $n$ & Media & $\sigma$ \\
\hline Básico & 133 & 30.86 & 6.262 \\
Medio & 131 & 31.56 & 6.162 \\
Integración & 124 & 30.90 & 6.076 \\
\hline
\end{tabular}

Tabla 3

Datos descriptivos de la variable aprendizaje, según el nivel académico y las áreas curriculares del programa académico de la carrera de arquitectura en la UANL $(N=388)$

\begin{tabular}{lclcc}
\hline $\begin{array}{c}\text { Nivel } \\
\text { académico }\end{array}$ & $n$ & \multicolumn{1}{c}{ Área curricular } & Media & $\sigma$ \\
\hline Básico & 133 & Diseño & 18.74 & 3.825 \\
& & Teórica Humanística & 17.68 & 3.813 \\
& Tecnología & 16.29 & 4.690 \\
& & Formación General & 16.69 & 6.262 \\
Medio & \multirow{2}{*}{131} & Diseño & \\
& & Teórica Humanística & 15.76 & 4.133 \\
& & Tecnología & 14.86 & 4.394 \\
& & Formación General & 17.42 & 4.156 \\
Integración & \multirow{2}{*}{124} & Diseño & 17.91 & 3.478 \\
& & Teórica Humanística & 17.07 & 3.906 \\
& & Tecnología & 16.29 & 4.102 \\
& & Formación General & 18.59 & 3.640 \\
\hline
\end{tabular}


estudiantes no conserva la tendencia alta que poseía los primeros semestres. Por el contrario, parece que algunos estudiantes bajan su nivel de autoestima al final de su carrera.

A continuación se presentan algunas tendencias porcentuales de las áreas disciplinares que conforman cada uno de los niveles académicos.

En el Nivel Básico, los resultados muestran que los estudiantes se perciben con cierta carencia de aprendizaje en lo concerniente al desarrollo de habilidades del pensamiento -como el análisis, la síntesis y la creatividad-, la aplicación de la topografía en sus proyectos, el conocimiento y la aplicación de los componentes de la metodología y el proceso de diseño, así como la fundamentación de sus proyectos de diseño con base en conceptos teóricos.

De acuerdo con los resultados descriptivos, en el Nivel Medio se destacan como áreas débiles las relacionadas con el área curricular de Tecnología, especialmente en la administración de proyectos y obras en construcción, instalaciones hidráulicas, sanitarias y de aire acondicionado; en el área Teórico-Humanística, los estudiantes se perciben con falta de conocimiento y comprensión de las corrientes de la arquitectura contemporánea en general, así como en la arquitectura mexicana y regional; asimismo los resultados muestran una fortaleza en el área de Formación General Universitaria, donde los estudiantes obtienen mayores puntuaciones.

En el Nivel de Integración, los estudiantes se perciben con una especial carencia de seguridad en los conocimientos adquiridos en el área curricular de tecnología. Particularmente en lo que se refiere a la utilización de los sistemas estructurales y mayormente en las actividades propias de la administración de proyectos y obras de construcción, así como en la capacidad para realizar análisis de factibilidad y viabilidad de proyectos arquitectónicos asociados al diseño urbano. Puede observarse una tendencia entre los estudiantes de percibirse con una falta de competencia como profesionales independientes que tomen la iniciativa de crear su propia empresa. En el mismo Nivel de Integración, en el área curricular de Diseño, los estudiantes perciben que tienen carencia hacia las actividades propias del diseño urbano y la representación profesional con medios digitales. Del mismo modo, en el área de TeóricoHumanística, los estudiantes se perciben con una falta de conocimiento en lo que se refiere a la utilización de una metodología durante el proceso del diseño y la capacidad de fundamentar y sustentar los proyectos arquitectónicos, con base en la teoría arquitectónica en sus funciones básicas de expresión, función y tecnología; es decir, en la aplicación y la transferencia de la teoría a la práctica profesional de la arquitectura. En todos los casos, las áreas que se han mencionado en donde se encontró una falta de capacidad o aprendizaje desde la percepción de los estudiantes, constituyen áreas de oportunidad para trabajar en los próximos planteamientos curriculares.

\section{Pruebas de hipótesis}

Este estudio tiene el objetivo de conocer la relación que existe entre la autoestima y el aprendizaje autopercibido de los estudiantes universitarios de arquitectura de la UANL, en el período agosto-diciembre de 2006. Con este propósito se consideraron las hipótesis nulas para los distintos niveles académicos -Básico, Medio y de Integración- y áreas curriculares que los conforman 


\section{CANTÚ HINOJOSA}

-Diseño, Teórico-Humanístico, Tecnología y Formación General Universitaria-. Para probar las hipótesis nulas se recurrió a la prueba de dependencia $r$ de Person, estableciendo como criterio de decisión un nivel de significación estadística de .05 .

Los resultados de las correlaciones entre la autoestima y el aprendizaje autopercibido se presentan en la Tabla 4. En ella se muestran los resultados de las pruebas de hipótesis. Se observa que la autoestima correlacionó significativamente con el aprendizaje autopercibido por los estudiantes del Nivel Medio en las áreas curriculares de Tecnología $(r=$ .292) y de Formación General $(r=.178)$, por lo que se desacreditan las hipótesis nulas formuladas para esas áreas y se sostienen las hipótesis de trabajo en donde se expone que existe una relación significativa entre las variables del estudio.

Tabla 4

Coeficientes de correlación y nivel de significatividad de la relación entre la autoestima y el aprendizaje autopercibido por niveles académicos y sus respectivas áreas curriculares $(N=388)$

\begin{tabular}{lclcc}
\hline $\begin{array}{c}\text { Nivel } \\
\text { académico }\end{array}$ & $n$ & \multicolumn{1}{c}{ Área curricular } & $r$ & $p$ \\
\hline Básico & \multirow{2}{*}{$\begin{array}{l}\text { Diseño } \\
\text { Teórica Humanística }\end{array}$} & .147 &. .013 & .092 \\
& & Tecnología & .072 & .409 \\
& & .018 & .835 \\
Medio & \multirow{2}{*}{131} & Formación General & & \\
& & Diseño & .107 & .222 \\
& & Teórica Humanística & .074 & .403 \\
& & Tecnología & $.292^{* *}$ & .001 \\
Integración & \multirow{2}{*}{124} & Formación General & $.178^{*}$ & .042 \\
& & & \\
& & Diseño & $.181^{*}$ & .044 \\
& & Teórica Humanística & .082 & .364 \\
& & Tecnología & .075 & .409 \\
& & Formación General & $.249^{* *}$ & .005 \\
\hline
\end{tabular}

$* * p<0.01 . \quad * p<0.05$.

En la submuestra de estudiantes del Nivel de Integración, los resultados muestran que la variable autoestima correlacionó significativamente con la del aprendizajeautopercibido, particularmente en las áreas curriculares de Diseño $(r=.181)$ y de Formación General universitaria $(r=.249)$, por lo que se desacreditan las hipótesis nulas formuladas para esas áreas y se sostienen las hipótesis de trabajo en donde se expone que existe una relación significativa entre las variables del estudio.

No se encontró correlación entre la autoestima y el aprendizaje autopercibido en los estudiantes del Nivel Básico, 
en ninguna de las áreas curriculares.

\section{Discusión}

Es evidente que la autoestima está relacionada con los procesos de aprendizaje en donde participa un marco complejo de variables. El presente estudio se centró en el análisis de la relación entre la autoestima y el aprendizaje, en particular manifestado desde la percepción de los estudiantes de arquitectura de la UANL en el período de agosto-diciembre de 2006, en los diferentes niveles académicos y en las áreas curriculares que los conforman.

Los resultados muestran que no se encontró correlación entre la autoestima y el aprendizaje autopercibido de los estudiantes del Nivel Básico en ninguna de las áreas curriculares del mismo programa educativo. Cabe mencionar que en este nivel académico los estudiantes están iniciando la carrera y es natural que presenten un buen nivel de autoestima, de acuerdo con la expectativa de haber iniciado el camino hacia una meta más de su vida, la de terminar una carrera profesional, pues, como señalan Bednar, Wells y Peterson (1995), la autoestima implica competencia, seguridad, dominio, logro, independencia y libertad. Sin embargo, aunque los estudiantes se perciben con cierta carencia de aprendizaje en lo concerniente al desarrollo de habilidades del pensamiento - como el análisis, la síntesis y la creatividad-, la aplicación de la topografía y la metodología del diseño en sus proyectos y la transferencia de los conceptos teóricos en los proyectos de diseño, estas habilidades autopercibidas no muestran una correlación significativa con la autoestima, por lo que se puede asumir que en ese nivel aún no influye en su percepción de lo que hasta ese momento de su carrera aceptan haber aprendido o no en las distintas áreas curriculares.

Por otra parte, los resultados muestran que la autoestima correlacionó significativamente con el aprendizaje percibido por los estudiantes del Nivel Medio en las áreas curriculares de Tecnología $(r=.292)$ y en la de Formación General Universitaria $(r=.178)$. De acuerdo con estos resultados, en el área de Tecnología, los estudiantes se perciben débiles en las áreas de administración de proyectos y obras en construcción, y en instalaciones hidráulicas, sanitarias y de aire acondicionado, por lo que éstas se presentan muy relacionadas con el nivel de autoestima. Se infiere que en la medida en que ellos se sientan más seguros de su capacidad para resolver problemas de éstas áreas, su autoestima se verá fortalecida, ya que la arquitectura no se puede ejercer profesionalmente con éxito cuando se cuenta con una carencia en los aspectos técnicos de la disciplina, tal como lo señala Palladino (1994), cuando menciona que el aspecto de confianza en sí mismo se da en una autoestima sana, que permite apreciar el valor e importancia propios, y tener el carácter para ser responsable de sí y de actuar responsablemente hacia los demás.

A su vez, los resultados muestran una fortaleza en el área de Formación General Universitaria, siendo ésta la que obtiene mayores puntuaciones. En la medida en que el estudiante siga fortaleciendo su competencia en está área de Formación General Universitaria, su nivel de autoestima será elevada debido a la correlación que presenta.

Con respecto al Nivel de Integración, también se observa que la autoestima correlacionó significativamente con el aprendizaje percibido por los estudiantes de este nivel en las áreas curriculares de 


\section{CANTÚ HINOJOSA}

Diseño y en la de Formación General.

Llama la atención la correlación particularmente en el área de diseño, ya que es el área que se considera la "columna vertebral" de la carrera de arquitecto. En ella se conjuntan todos los conocimientos adquiridos en las otras áreas curriculares y de especial manera en los últimos semestres, como lo es en el Nivel de Integración.

Aunque los resultados muestran una correlación significativa con el área de diseño, los estudiantes allí también perciben que tienen carencia hacia las actividades propias del diseño urbano y la representación profesional de los proyectos arquitectónicos con medios digitales. Por otra parte, en el área TeóricoHumanística los estudiantes se perciben con una falta de conocimiento en lo referente a la utilización de una metodología durante el proceso del diseño y la capacidad de fundamentar y sustentar los proyectos arquitectónicos; es decir, en lo concerniente a la aplicación y la transferencia de la teoría a la práctica profesional de la arquitectura.

Estos resultados de alguna manera ponderan la correlación significativa con la variable autoestima. Sin embargo, el valor de ésta sería aún más alto si, aunado a lo anterior, los estudiantes se percibieran con mayor competencia en las áreas en que se perciben débiles en cuanto a su capacidad académico-profesional, coincidiendo con las investigaciones de González Pumariega Solís et. al. (1994), que ponen de manifiesto que la implicación activa del sujeto en el proceso de aprendizaje aumenta cuando confía en sus propias capacidades y tiene altas expectativas de autoeficacia.

Se observa en los estudiantes que están próximos a graduarse y en algunos egresados la tendencia a percibirse con una falta de competencia como profesionales independientes que tomen la iniciativa de crear su propia empresa. Esto podría representar una carencia en la seguridad personal y la autoestima al sentir una falta de competencia, aunque quizás también se perciben con la carencia de otros recursos necesarios para que se dé esa actividad, entre ellos la motivación. Según Gecas (1982), las cosas que los individuos escogen y la forma en que lo hacen parecen estar en dependencia de su propia autoestima, que está correlacionada con el comportamiento funcional y la satisfacción en la vida.

Es necesario mencionar el impacto que tendrían otros factores que confluyen en esta etapa de la formación académica y el inicio del ejercicio profesional, tales como desempeñar un servicio social poco atractivo y en ocasiones sin relación con los conocimientos y las habilidades aprendidas durante el transcurso de su carrera y carecer de los recursos financieros para llevar a cabo el trámite de la titulación, los cuales podrían fortalecer o debilitar el autoconcepto del individuo al término de sus estudios profesionales.

Conclusiones

La principal contribución del presente estudio es la demostración de que el aprendizaje -desde la percepción de los estudiantes-interacciona con el nivel de autoestima de cada estudiante de acuerdo con el nivel que cursa. De acuerdo con las pruebas de hipótesis, de las cuales se obtienen los resultados de este estudio, se concluye en términos generales, que existe una correlación entre la autoestima y el aprendizaje autopercibido, especialmente en los Niveles Medio y de Integración del programa académico de arquitectura de la UANL. 
Particularmente esta correlación se presentó en las áreas curriculares de Tecnología y Formación General Universitaria para el Nivel Medio y en las de Diseño y Formación General Universitaria para el Nivel de Integración. No se encontró correlación de la autoestima y el aprendizaje autopercibido de los estudiantes del Nivel Básico en ninguna de las áreas curriculares del mismo programa educativo. Dado que la correlación se observó en los niveles que constituyen las etapas finales de la carrera, todo parece indicar que, a medida que el estudiante se acerca a la conclusión de su formación académica y profesional es cuando se manifiesta una correlación directa de su autoestima con su percepción del aprendizaje. Esto representa una implicación futura para la atención de esta variable en la formación académico-profesional de los estudiantes universitarios, ya que al sentirse seguros de lo que son capaces de hacer profesionalmente, manifiestan mayor autoestima y viceversa. Es de esperarse que en la medida que la autoestima esté mayormente desarrollada con una tendencia alta le ayudará al estudiante a desempeñarse con mayor éxito en su vida personal y profesional de una manera integral.

La correlación autoestima-aprendizaje autopercibido está inmersa en el proceso de enseñanza y de aprendizaje. Debido a ello se podría convertir en uno de los desafíos más complejos que enfrenta la educación superior en la actualidad. El aprendizaje manifestado en la formación profesional, muchas de las veces está influido por el comportamiento, las percepciones personales, las interrelaciones, el contexto, la motivación, el autoconcepto y el nivel de autoestima, entre otros factores. En este sentido, la relación entre los niveles de aprendizaje y los aspectos particulares del individuo lleva a pensar en la importancia de que los procesos de enseñanza y de aprendizaje que incluyen tanto a alumnos, docentes e institución, consideren modelos curriculares que atiendan la formación integral además de la disciplinar. Se recomienda apoyar las propuestas para la realización de otros estudios en esa dirección.

Con el propósito de que los resultados de este estudio sean de utilidad, se sugiere tomar en cuenta como un principio rector la relación e integración del desarrollo de la autoestima en los procesos de aprendizaje y la formación de competencias - conocimientos, habilidades, actitudes y valores-, en los estudiantes de arquitectura de la UANL, para planear y coordinar la práctica docente y el programa académico de la carrera de arquitecto en función de los aspectos que favorezcan el mejoramiento del rendimiento académico y profesional en general $y$, particularmente, del desempeño del diseño arquitectónico, considerado como la actividad central del arquitecto.

\section{Referencias}

Abbagnano, N. (1994). Diccionario de filosofia ( $11^{\mathrm{a}}$ ed.). México: Fondo de Cultura Económica.

Bednar, R. L., Wells, M. G. y Peterson, S. R. (1995). Self-esteem: Paradoxes and innovations in clinical theory and practice (2a. ed.). Washington: American Psychological Association.

Beltrán, J. (1987). Psicología de la educación. Madrid: Eudema.

Boersma, F. J. y Chapman, J. W. (1985). Manual of the Studen's Perception of Ability Scale. Edmonton, Canada: University of Alberta.

Bruner, J. (1996). The culture of education. London: Harvard University Press.

Burns, R. B. (1980). Introductory psychology. Lancaster: MTP Press.

Burns, R. B. (1990). El autoconcepto: teoría, medición, desarrollo y comportamiento. 


\section{CANTÚ HINOJOSA}

Bilbao: EGA.

Byrne, B.M. y Gavin, D.A.W. (1996). The Shavelson model revisited: Testing for the structure of academic self-concept across pre-early, and late adolescents. Journal of Educational Psychology, 88(2), 215-228.

Chapman, J. W. y Lambourne, R. (1990). Some antecedents of academic self-concept: A longitudinal study. The British Journal of Educational Psychology, 60, 142-152.

Coll, C. (1992). Desarrollo psicológico y educación (Vol. 2). Madrid: Alianza.

Combs, A. W. (1974). Educational accountability: A humanistic perspective. San Francisco: Shields.

Cotton, J. W. (1989). Antecedentes históricos de la teoría del aprendizaje. Enciclopedia Internacional de la Educación. Barcelona: MEC y Vicens-Vives.

Covington, J. y Omelich, C. (1988). Effort: The double edged sword in school achievement. Journal of Educational Psychology, 71, 169182.

Covington, M. V. (1984). Strategic thinking and the fear of failure. En J. Segal, S. Chipman y R. Glaser (Eds.), Thinking and learning skills: Vol. 1: Relating instruction to basic research (pp. 389-415). Hillsdale. NJ: Erlbaum.

Covington, M. (1992). Making of the grade. Cambridge: Cambridge University Press.

García Torres, B. (1983). Análisis y delimitación del constructo autoestima.. Madrid: Universidad Complutense de Madrid.

Gecas, V. (1982). The self concept. Annual Review of Sociology, 8(3), 1-33.

González-Pumariega Solís, S., García Rodríguez, M. S., García garcía, S. I., Núñez Pérez, J. C. y González-Pienda García, J. A. (1994). Estrategias de aprendizaje en alumnos de 10 a 14 años y su relación con los procesos de atribución causal, el autoconcepto y las metas de estudio. Revista Galega de Psicopedgoxía, 7(10-11), 219-242.

Goodwin, S. G. (1999). Developing self-esteem in physical education. Physical Educator, 56(4), 210-215.

Harter, S. (1982). The Perceived Competence Scale for Children. Child Development, 53, 87-97.

Harter, S. (1985a). Competence as a dimension of self-evaluation: Toward a comprehensive model of self-worth. En R. Leahy (Ed.), The development of the self (pp. 55-121). San Diego, CA: Academic Press.

Harter, S. (1985b). Manual for the Self-Percep- tion Profile for Children: Revision of the Perceived Competence Scale for Children. Manuscrito no publicado. Denver, CO: University of Denver.

Harter, S. (1986). Comparisons of alcohol and other drug problems among Minnesota adolescents. Archives of Pediatrics and Adolescent Medicine, 149, 137-144.

Harter, S. (1999). Causes, correlates and the functional role of global self-worth: A lifespan perspective. En J. Kolligian y R. Sternberg (Eds.), Perception of competence and incompetence across the life-span (pp. 6797). New Haven: Yale University Press.

Helmke, A. y Van Aken, M. A. (1995). The casual ordering of academic achievement and selfconcept of ability during elementary school: A longitudinal study. Journal of Educational Psychology, 87, 624-637.

Hernández, P. (1991). Psicología de la instrucción. México: Trillas.

Hernández Sampieri, R., Fernández Collado, C. y Baptista Lucio, P. (2006). Metodología de la investigación $\left(4^{\mathrm{a}}\right.$ ed.). México: Mc. Graw Hill.

Markus, I. M. y Nurius, P. (1986). Possible selves. American Psuchologist, 41, 954-969.

Marsh, H. W. (1986). The hierarchical structure of self-concept in the perception of others. Journal of Educational Measurement, 24, 17-19.

Marsh, H. W. (1988). Self-Description Questionnaire: $S D Q$ manual and research monograph. San Antonio: The Psychological Corporation.

Marsh, H. W. (1990a). Causal ordering of academic self-concept and academic achievement: A multiwave, longitudinal panel analysis. Journal of Educational Psychology, 82, 646656.

Marsh, H. W. (1990b). Causal ordering of academic self-concept on academic achievement: A reanalysis of Newman (1984). Journal of Experimental Education, 56, 100-103.

Marsh, H.V., Parker, J.W. y Smith, I. D. (1983). Preadolescent self-concept: Its relations to self-concept as inferred by teachers and to academic ability. British Journal of Educational Psychology, 53, 60-78.

McCombs, B. L. (1991). Motivation and lifelong learning. Educational Psychologist, 26(2), 117-127.

McCombs, B. L.y Marzano, R. J. (1990). Putting the self in self-regulating learning: The self as agent in integrating will and skills. Educational Psychologist, 25(1), 51-69.

McCombs, B. L. y Whisler, J. S. (1997). The 


\section{AUTOESTIMA Y PERCEPCIÓN DEL APRENDIZAJE}

learner-centered classroom and school. San Francisco: Jossey-Bass.

Melendo, T. (2006). Felicidad y autoestima. Madrid: Ediciones Internacionales Universitarias.

Oñate y García, P. (1995). Autoconcepto. En J. Beltrán Llera y J. A. Bueno Álvarez (Eds.), Psicología de la educación (pp. 212-226). Barcelona: Marcombo.

Palladino, C. (1994). Autoestima para estudiantes. México: Iberoamérica.

Purkey, W. W. (1970). Self-concept and school achievement. Englewood Cliffs, NJ: Prentice Hall.

Purkey, W. W. y Novak, J. (1984). Inviting school success. Belmont, CA: Wadsworth.

Ribeiro, L. (2006). Desarrollo humano. Barcelona: Odisea.

Román Pérez, M. y Díez López, E. (2000). Aprendizaje y currículum: diseños curriculares aplicados. Buenos Aires: Novedades Educativas.

Shavelson, R. J. y Bolus, R. (1982). Self-concept:
The interplay of theory and methods. Journal of Educational Psychology, 74, 3-17.

Shavelson, R. J., Hubner, J. J. y Stanton, G. C. (1976). Validation of construct interpretations. Review of Educational Research, 46 , 407-441.

Skaalvik, E. M. y Hagtvet, K. A. (1990). Academic achievement and self-concept: An analysis of causal predominance in a developmental perspective. Journal of Personality and Social Psychology, 58(2), 292-307.

Trianes, M. (1996). Psicología de la educación para profesores. Pirámide: Madrid.

Recibido: 19 de mayo de 2007

Revisado: 25 de junio de 2007

Aceptado: 14 de julio de 2007 